\title{
Linear polarization in forbidden lines of the T Tauri star RY Tauri
}

\author{
H. Akitaya ${ }^{1,7}$, Y. Ikeda ${ }^{2}$, K. S. Kawabata ${ }^{3}$, K. Matsuda ${ }^{4}$, A. Okazaki ${ }^{5}$, and M. Seki ${ }^{6}$ \\ ${ }^{1}$ ELT Project Office, National Astronomical Observatory of Japan, Osawa 2-21-1, Mitaka, Tokyo 181-8588, Japan \\ e-mail: hiroshi.akitaya@nao.ac.jp \\ 2 Photocoding, Higashi-Hashimoto 3-16-8-101, Sagamihara, Kanagawa 229-1104, Japan \\ 3 Astrophysical Science Center, Hiroshima University, 1-3-1 Kagamiyama, Higashi-Hiroshima, Hiroshima 739-8526, Japan \\ 4 Nishi-Harima Astronomical Observatory, Sayo-cho, Hyogo 679-5313, Japan \\ Department of Science Education, Gunma University, Aramaki, Maebashi, Gunma 371-8510, Japan \\ 6 Astronomical Institute, Graduate School of Science, Tohoku University, Aramaki, Aoba, Sendai 980-8578, Japan \\ 7 Visiting Astronomer, Okayama Astrophysical Observatory, National Astronomical Observatory of Japan, Asakuchi, \\ Okayama 719-0232, Japan
}

Received 2 November 2008 / Accepted 6 March 2009

\section{ABSTRACT}

\begin{abstract}
We performed high-dispersion spectropolarimetry for the T Tauri star RY Tauri and measured linear polarization in seven forbidden lines of [OI] $\lambda \lambda 5577,6300,6364$, [NII] $\lambda 6583$, [SII] $\lambda \lambda 6716,6731$, and [FeII] $\lambda 7155$. This is the first high-dispersion spectropolarimetry for forbidden lines in T Tauri stars. We successfully detected intrinsic polarization in the $[\mathrm{OI}] \lambda 6300$ line. The intrinsic [OI] $\lambda 6300$ polarization corrected for the foreground polarization was derived as $p_{[\mathrm{OI}]}=1.17 \pm 0.54 \%$ and $\theta_{[\mathrm{OI}]}=107^{\circ} \pm 13^{\circ}$, whereas the polarization of the nearby continuum was $p_{\text {cont }}=2.4-3.1 \%$ and $\theta_{\text {cont }}=2^{\circ}-11^{\circ}$. The position angle of [OI] $\lambda 6300$ polarization is compared with the circumstellar structures found by previous studies. It is nearly perpendicular to the disk long-axis and parallel to the optical $\mathrm{H} \alpha$ jet. Both the perpendicularity and parallelism potentially suggest relatively axisymmetric distribution of the [OI] 26300 emitting region and surrounding scattering medium. We constructed a simplified scattering model composed of a point source on a jet axis as an [OI] $\lambda 6300$ emitter and a flat disk with an inner hole as a scatterer. Applying the observed polarization to the model suggests that the $[\mathrm{OI}] \lambda 6300$ emission emerges close to the central star with a possible separation of less than a few $\times 0.1 \mathrm{AU}$.
\end{abstract}

Key words. stars: pre-main sequence - circumstellar matter - polarization - techniques: polarimetric - techniques: spectroscopic stars: individual: RY Tauri

\section{Introduction}

T Tauri stars (TTSs) are low-mass (less than about two solar masses) pre-main sequence stars at a stage of evolution during which the central star is contracting and gathering matter through its circumstellar disk. Outflows of TTSs such as jets and winds play an important role in the evolution of the star itself and of the circumstellar disk because they regulate mass and angular momentum of the star and disk system. However, the launching mechanism of the outflows, for which the X-wind model (Shang et al. 2007) and the disk wind model (Pudritz et al. 2007) have been proposed, is still controversial. To clarify the outflow mechanism, it is crucial to probe the inner structure of the outflows close to the central star on the sub-AU scale where the flow emerges.

Forbidden emission lines (FELs) are suitable for probing outflows of TTSs because they usually trace only outflows. Furthermore, their optically-thin and absorption-free properties are very helpful in interpreting the lines physically. Permitted lines such as Balmer lines of hydrogen also trace outflows, but their interpretation is complicated in general because those lines often trace infall phenomena as well and absorption features varying in short time scales also get mixed in the spectrum.

Since the 1990s, much work has been done by highdispersion spectrometry as well as by long-slit spectrometry for FELs in TTSs (Hamann 1994; Hirth et al. 1994a,b; Hartigan et al. 1995; Hirth et al. 1997; Bacciotti et al. 2000). They revealed both the velocity and spatial distribution of forbidden emission lines in TTSs with resolutions of a few $\times 10 \mathrm{~km} \mathrm{~s}^{-1}$ and $\sim 0.1$ arcsec, respectively, and established general insights into the structure of jets and winds around TTSs (see Ray et al. 2007). However, higher spatial resolution is still required to approach the launching region of outflows close to the central star. Recently, new observational efforts such as spectro-astrometry (Takami et al. 2001) and long slit spectroscopy with an adaptive optics (Pyo et al. 2003, 2006) have been made to reveal the circumstellar structure of TTSs down to AU scales.

High-dispersion spectropolarimetry of emission lines is an alternative tool for investigating the structure around the emitting region close to the central star on a sub-AU scale because these lines are expected to show linear polarization through light scattering in the circumstellar structures and to give geometrical information on the distribution of emitters and scatterers. Vink et al. (2003, 2005) measured linear polarization in ten TTSs at an $\mathrm{H} \alpha$ line. They presented remarkable polarization changes within the line, and discussed the size of the emitting region and of the disk inner hole. Their work has been the first and epochal polarimetric study on an emission line of TTSs that shed light on the inner disk structure directly taking part in the mass inflow around the central star. On FELs, however, no intensive spectropolarimetric study has been reported to probe the outflows in TTSs. Thus we performed high-dispersion spectropolarimetry for one of the TTSs, RY Tauri, and measured linear polarization of FELs in TTSs for the first time. 
Table 1. Observation journal of high-dispersion $(R=7000)$ spectropolarimetry for RY Tauri.

\begin{tabular}{cccccc}
\hline \hline $\begin{array}{c}\text { Date } \\
\text { (HST) }\end{array}$ & JD & $\begin{array}{c}\text { Exposure time } \\
{[\mathrm{s}]}\end{array}$ & $\begin{array}{c}\text { Seeing } \\
{[\operatorname{arcsec}]}\end{array}$ & Weather & $\begin{array}{c}\text { Spectral range } \\
{[\AA]}\end{array}$ \\
\hline March 1, 2002 & 2452335.76 & 7200 & 1.0 & Fair & $5660-8620$ \\
March 3, 2002 & 2452337.74 & 8640 & 0.8 & Fair & $5660-8620$ \\
October 26, 2002 & 2452575.00 & 10800 & $0.4-0.5$ & Fair & $5320-8600$ \\
\hline
\end{tabular}

RY Tauri is one of the brightest, most frequently observed classical $\mathrm{T}$ Tauri stars with a mass of $\sim 1.63 M_{\odot}$, age of $\log t(\mathrm{yr}) \sim 6.20$ (Hartigan et al. 1995) and spectral type of G1-G3IV (Holtzman et al. 1986; Petrov et al. 1999). Though deduced parameters differ slightly among authors, the object evidently belongs to the group of classical T Tauri stars.

In Sect. 2, we present high-dispersion spectropolarimetry for RY Tauri and the estimation of the foreground polarization toward the object. In Sect. 3, we show the results on polarization measurements of FELs in RY Tauri and report the significant detection of linear polarization in an [OI] $\lambda 6300$ emission line. We discuss the emitting region of the [OI] $\lambda 6300$ line by comparing the detected polarization with the scattering model in Sect. 4 . We summarize the results and discussions in Sect. 5.

\section{Observation and data reduction}

\subsection{High-dispersion spectropolarimetry for RY Tauri}

We obtained high-dispersion linear polarization spectra of RY Tauri using the echelle spectropolarimeter LIPS (Ikeda et al. 2003) attached on a Cassegrain focus of the University of Hawaii 2.2-m telescope with an $f / 31$ secondary mirror at Mauna Kea Observatory. Observations were carried out at three epochs: March 1, 2002; March 3, 2002; and October 26, 2002. Using an entrance slit of $0.2 \mathrm{~mm}$ width, which corresponds to 0.6 arcsec on the sky, and a $300 \mathrm{~g} / \mathrm{mm}$ grating as a cross disperser, we obtained spectra of intensity and linear polarization at the spectral resolution of $R \sim 7000$ or velocity resolution of $\sim 45 \mathrm{~km} \mathrm{~s}^{-1}$ over a spectral range from about $5300 \AA$ to $8600 \AA$ on an EEV $2 \mathrm{~K} \times 2 \mathrm{~K}$ pixels $\mathrm{CCD}$. An observation journal is summarized in Table 1.

We obtained either thirteen or fourteen orders echelle spectra at each exposure. Each order consists of a pair of two spectra that are perpendicularly polarized rays split by a Wollaston prism after being modulated by a superachromatic half-wave plate. We exposed the object at four different position angles of the half-wave plate from $0^{\circ}$ to $67.5^{\circ}$ with a $22.5^{\circ}$ step. The data was reduced using both $I R A F^{1}$ (Tody 1993), and the C and Java software originally developed by our group. After the usual reduction procedures including bias subtraction, bad pixels correction, cosmic-ray subtraction, flattening, extraction of the spectra and wavelength coordinate allocation, we obtained eight independent spectra that consist of pairs of perpendicularly polarized spectra at four position angles of the half-wave plate. We finally reduced those spectra according to the procedure described in Kawabata et al. (1999), and obtained Stokes $I, Q$, and $U$ spectra, which can be converted into normalized Stokes parameters of $q \equiv Q / I$ and $u \equiv U / I$, or polarization degree $p$ and position angle $\theta$ using the relations $p=\left[(Q / I)^{2}+(U / I)^{2}\right]^{1 / 2}$ and $\theta=(1 / 2) \arctan (U / Q)$.

${ }^{1}$ IRAF is distributed by the National Optical Astronomy Observatories, which are operated by the Association of Universities for Research in Astronomy, Inc., under cooperative agreement with the National Science Foundation.
The instrumental polarization was measured by observing unpolarized standard stars each night. They were below about $0.05-0.15 \%$ and stable over the whole wavelength range. We subtracted the instrumental polarization averaged over the unpolarized standard stars at each observing run from observed polarization spectra. Our instrument avoids the problems of "ripples" in a polarimetric spectrum (Harries \& Howarth 1996) by using an AstroPribor Super-Achromatic Zero-Order Waveplate as a half-wave plate (Ikeda et al. 2003; Samoylov et al. 2004). We achieved a polarimetric accuracy of better than $0.1 \%$ without any corrections for ripples.

The instrumental origin of polarization position angle on the sky was determined to an accuracy of about 0.25 degrees by measuring strongly polarized standard stars selected from Schmidt et al. (1992). Both the wavelength dependency of instrumental depolarization and that of the optical axis of the halfwave plate were determined from the observation of artificially polarized stars through a Glan-Taylor prism followed by the entrance slit. The typical depolarization factor, which is to be applied to the observed polarimetric spectra, was less than $0.5 \%$ of the observed polarization. The optical axis of the half wave plate was determined with an accuracy of 0.05 degrees.

The wavelength coordinate of each spectrum was determined using the emission lines of a Th-Ar arc lamp. It was converted to the velocity coordinate at rest frame on the object using the deduced heliocentric radial velocity of $+14.8 \pm 1.6 \mathrm{~km} \mathrm{~s}^{-1}$, measured from a peak velocity of a photospheric LiI $\lambda 6708$ absorption line, which is consistent with the previous measurements (Hartmann et al. 1986; Hartmann \& Stauffer 1989; Petrov et al. 1999). The final accuracy of radial velocity was within several $\mathrm{km} \mathrm{s}^{-1}$.

As for [OI] forbidden emission lines, we subtracted telluric airglow emission mixed in the observed spectra. The airglow spectrum was estimated from the nearby sky portions of the exposed spectrum. The intensity of the airglow was $5-10 \%$ of the total observed intensity at [OI] $\lambda 6300,6-15 \%$ at $[\mathrm{OI}] \lambda 6364$, and $34 \%$ at $[\mathrm{OI}] \lambda 5577$, respectively.

Spectropolarimetric spectra of the [OI] $\lambda 6300$ line are shown in Fig. 1 as an example.

\subsection{Veiling}

We estimated the veiling effect in RY Tauri by measuring an equivalent width of the photospheric LiI $\lambda 6708$ absorption line. The equivalent widths at three epochs were $0.289 \pm 0.007 \AA$, $0.288 \pm 0.006 \AA$, and $0.255 \pm 0.009 \AA$, respectively, and we deduced the veiling as $-0.07 \pm 0.07,-0.06 \pm 0.07$, and $0.06 \pm 0.07$, respectively, by applying the intrinsic equivalent width of photospheric LiI line of $0.27 \pm 0.06 \AA$ measured by Basri et al. (1991). Since practically no significant veiling effect was detected, we neglect the effects of the veiling continuum from the hot gas on the photosphere hereafter. 


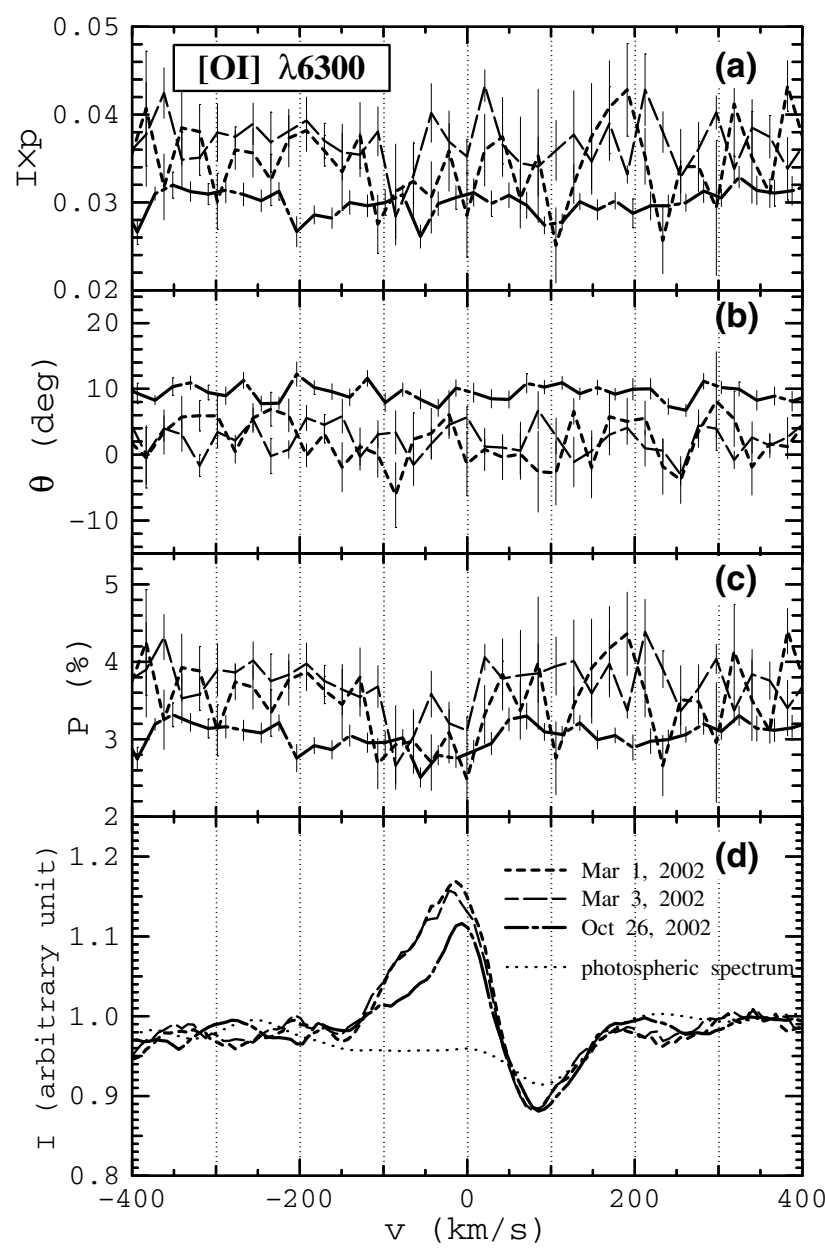

Fig. 1. Observed intensity and linear polarization spectra of an [OI] $\lambda 6300$ line. Spectra of a) polarized flux, b) position angle, c) polarization degree, and d) intensity, at three epochs: March 1, 2002 (dashed line), March 3, 2002 (long-dashed line), and October 26, 2002 (chain line). The foreground interstellar polarization, which is estimated as $p \sim 0.7 \%$ at $\theta \sim 6^{\circ}$ (see Sect. 2.4), is not subtracted. The bump of the polarization degree across the line is seen without any changes of the position angle because the nearly unpolarized line emission, which consists of the intrinsic polarization of the line itself and the foreground polarization, is overlapping the strongly polarized continuum (see Sect. 3.3). The estimated photospheric spectrum is also shown in the bottom panel (dotted line).

\subsection{Stokes spectra for emission lines}

The observed intensity profile is a blend of the forbidden emission line itself and the underlying photospheric continuum. We reduced the intensity and linear polarization Stokes spectra of each emission line by separating the underlying spectra on the simple assumption as follows:

$I_{\text {line }}(\lambda)=I_{\mathrm{obs}}(\lambda)-I_{\mathrm{ph}}(\lambda)$,

$Q_{\text {line }}(\lambda)=Q_{\mathrm{obs}}(\lambda)-q_{\mathrm{ph}} I_{\mathrm{ph}}(\lambda)$,

$U_{\text {line }}(\lambda)=U_{\text {obs }}(\lambda)-u_{\mathrm{ph}} I_{\mathrm{ph}}(\lambda)$,

where the subscripts "obs", "line", and "ph" represent the values from the observation, emission line, and underlying photospheric continuum, respectively. Parameters $q_{\mathrm{ph}}$ and $u_{\mathrm{ph}}$ are normalized Stokes parameters of a photospheric component measured at the nearby continuum.
A photospheric spectrum $I_{\mathrm{ph}}$ is not flat in general but contains photospheric absorption features. Spectral type of RY Tauri was determined as G1-G3 IV by the previous high-dispersion spectroscopy (Holtzman et al. 1986; Petrov et al. 1999). Montes et al. (1999) presented a digitized high-dispersion spectral atlas of late type stars taken with the spectral resolution of $R=12000$. We applied their spectrum of either HD 67228 (G1IVb) or HD 196755 (G2IV) according to their wavelength coverage in the atlas as a reference of the photospheric spectrum $I_{\mathrm{ph}}(\lambda)$ of RY Tauri, after tuning the spectral resolution to that of LIPS and making a correction for the rotational broadening by adopting $v \sin i=52 \mathrm{~km} \mathrm{~s}^{-1}$ of RY Tauri (Bouvier 1990; Hartmann \& Stauffer 1989; Petrov et al. 1999). The accuracy of coincidence between the estimated embedded photospheric spectrum and observed spectrum was $1-1.5 \% \mathrm{rms}$ for typical spectral regions.

For an [NII] $\lambda 6583$ line, a wing of the $\mathrm{H} \alpha$ line also underlies the line. The wing was assumed to be so smooth that its influence is correctly subtracted by the method described above.

We identified forbidden lines of [OI] $\lambda \lambda 5577,6300,6364$, [SII] $\lambda \lambda 6716,6731,[\mathrm{NII}] \lambda 6583$ and [FeII] $\lambda 7155$ in the intensity spectra of RY Tauri. The deduced intensity profiles after subtracting an underlying continuum spectrum are shown in Fig. 2.

\subsection{Estimation of the foreground polarization toward RY Tauri}

To investigate intrinsic polarization of the object itself, the precise determination and subtraction of its foreground interstellar polarization is crucial. We estimated the foreground polarization toward RY Tauri by a series of low-resolution spectropolarimetries for carefully selected field stars around RY Tauri.

We selected five field stars based on two criteria: a sufficiently small angular distance from RY Tauri not exceeding about 1.5 degrees on the sky and availability of a trigonometric parallax measurement by the Hipparcos satellite (ESA 1997). We measured their interstellar linear polarization spectrum between $3600 \AA$ to $9000 \AA$ at the spectral resolution of $R \sim 60$ $(\Delta \lambda \sim 100 \AA)$ using the low-resolution spectropolarimeter HBS (Kawabata et al. 1999) attached on either the 91-cm telescope of Dodaira Astronomical Observatory (DAO) or the 91-cm telescope of Okayama Astrophysical Observatory (OAO). The selected field stars and the observation journal are summarized in Table 2.

For a measured spectrum of polarization degree, we applied the empirical Serkowski curve (Serkowski et al. 1975; Whittet et al. 1992) given by

$p(\lambda)=p_{\max } \exp \left[-K \ln ^{2}\left(\frac{\lambda_{\max }}{\lambda}\right)\right]$

where

$K=1.66\left(\lambda_{\max } / 10000 \AA\right)+0.01$,

and deduced the peak polarization degree $p_{\max }$ and the wavelength $\lambda_{\max }$ in angstrom at which polarization degree has a peak. We assumed a linear function for the position angle spectrum in consideration of their slight variation with wavelength. We fitted the function

$\theta(\lambda)=\theta^{\prime} \cdot(\lambda-5500 \AA)+\theta_{5500}$,

and deduced the position angle at $5500 \AA\left(\theta_{5500}\right)$ and the gradient of position angle against wavelength $\left(\theta^{\prime}\right)$. The parameters derived for each object are summarized in Table 3. 


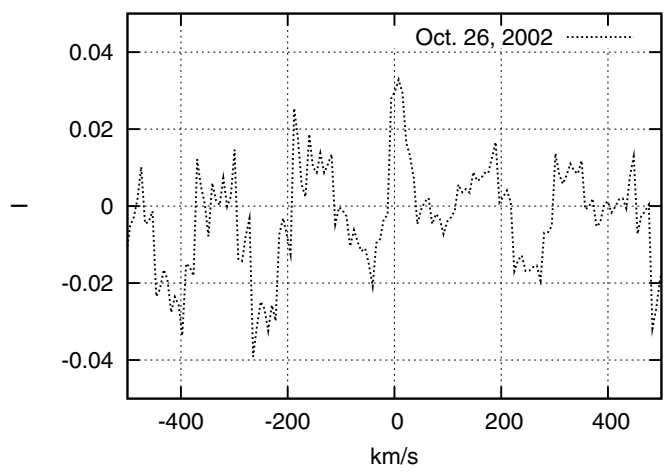

(a) $[\mathrm{OI}] \lambda 5577$

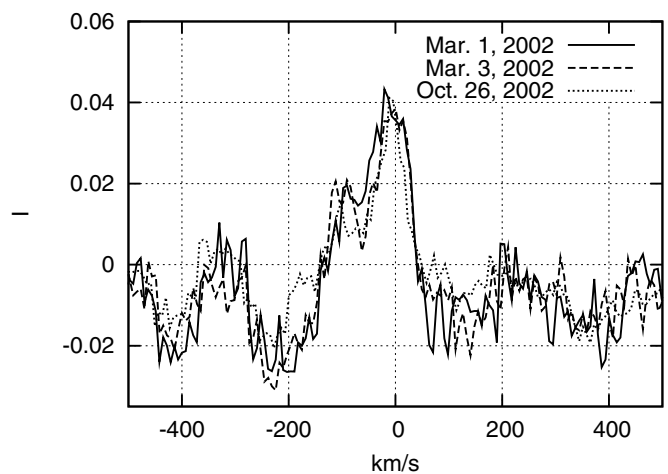

(c) $[\mathrm{OI}] \lambda 6364$

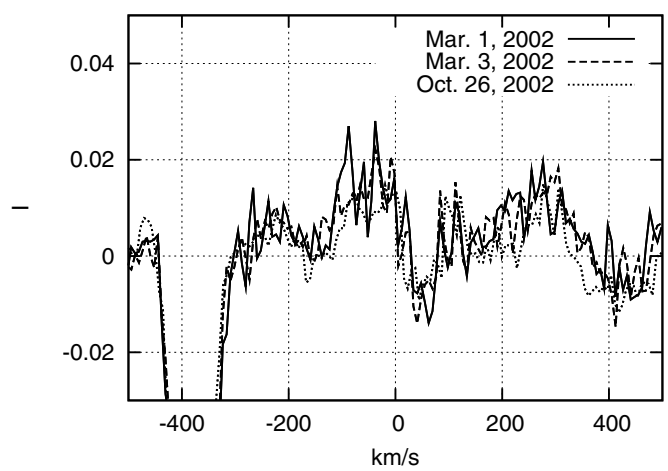

(e) $[\mathrm{SII}] \lambda 6716$

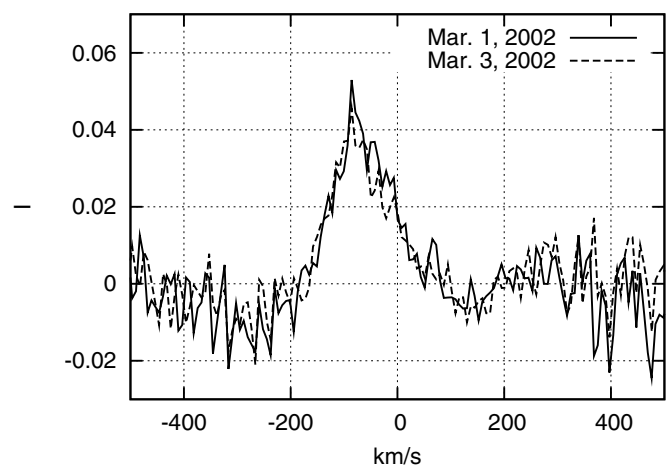

(g) $[\mathrm{FeII}] \lambda 7155$

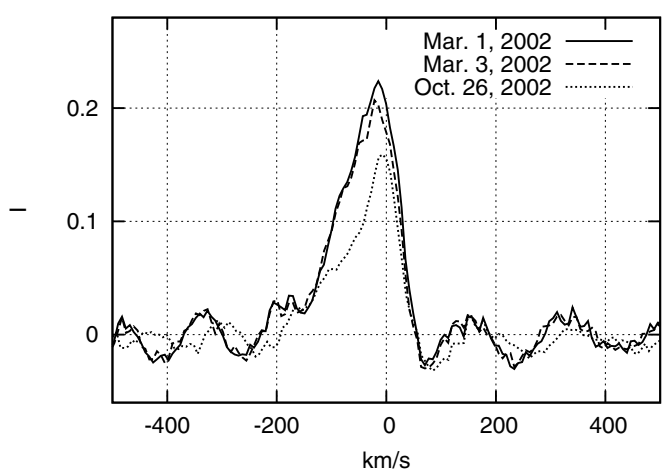

(b) $[\mathrm{OI}] \lambda 6300$

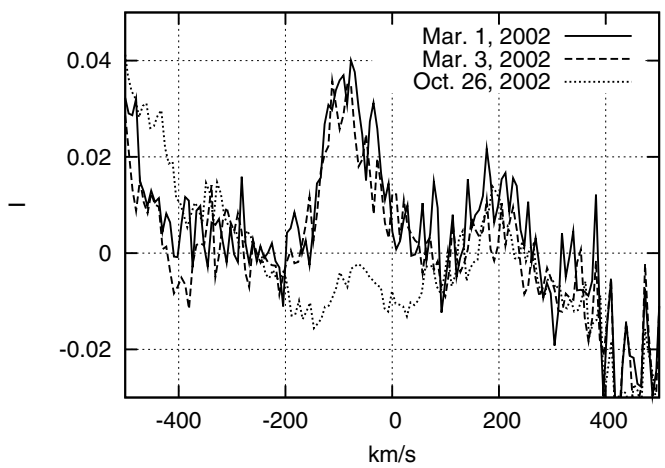

(d) $[\mathrm{NII}] \lambda 6583$

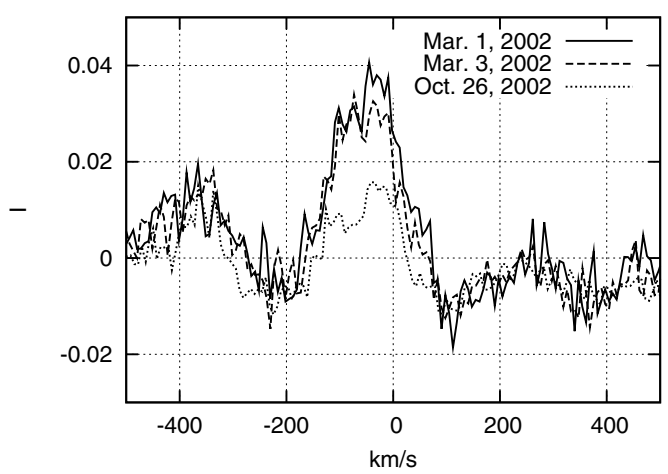

(f) $[\mathrm{SII}] \lambda 6731$

Fig. 2. Intensity profiles of forbidden emission lines. A normalized photospheric spectrum was subtracted from the raw spectrum normalized at continuum level. 
Table 2. Observation journal of low-resolution spectropolarimetry of the field stars around RY Tauri.

\begin{tabular}{ccccccc}
\hline \hline Object & $\begin{array}{c}m_{\mathrm{V}} \\
{[\mathrm{mag}]}\end{array}$ & RA (2000) & Dec (2000) & Date & Telescope & $\begin{array}{c}\text { Exposure time } \\
{[\mathrm{s}]}\end{array}$ \\
\hline HIP 20728 & 9.64 & $04^{\mathrm{h}} 26^{\mathrm{m}} 31^{\mathrm{s}} .02$ & $29^{\circ} 15^{\prime} 45^{\prime \prime} 52$ & Feb. 9, 2000; Mar. 12, 2000 & DAO 91 cm & 9400 \\
HIP 20250 & 4.97 & $04^{\mathrm{h}} 20^{\mathrm{m}} 21^{\mathrm{s}} .21$ & $27^{\circ} 21^{\prime} 02^{\prime \prime} 73$ & Nov. 16, 1999; Jan. 3, 2000 & DAO 91 cm & 2640 \\
HIP 20262 & 7.93 & $04^{\mathrm{h}} 20^{\mathrm{m}} 37^{\mathrm{s}} .78$ & $28^{\circ} 53^{\prime} 31^{\prime \prime} 24$ & Jan. 1, 2000 & DAO 91 cm & 3520 \\
HIP 20324 & 7.21 & $04^{\mathrm{h}} 21^{\mathrm{m}} 15^{\mathrm{s}} .26$ & $27^{\circ} 21^{\prime} 00^{\prime} .85$ & Nov. 24, 2000 & OAO 91 cm & 1600 \\
HIP 20663 & 8.17 & $04^{\mathrm{h}} 25^{\mathrm{m}} 38^{\mathrm{s}} .26$ & $29^{\circ} 31^{\prime} 36^{\prime \prime} 17$ & Dec. 2, 2000 & OAO 91 cm & 2560 \\
\hline cf. RY Tauri (HIP 20387) & & $04^{\mathrm{h}} 21^{\mathrm{m}} 57^{\mathrm{s}} .41$ & $28^{\circ} 26^{\prime} 35^{\prime \prime} 60$ & & & \\
\hline
\end{tabular}

Table 3. Measured interstellar polarization parameters of the field stars and estimated foreground polarization toward RY Tauri.

\begin{tabular}{ccccccc}
\hline \hline Object & $\begin{array}{c}\text { Parallax } \\
{[\mathrm{mas}]}\end{array}$ & $\begin{array}{c}\text { Distance } \\
{[\mathrm{pc}]}\end{array}$ & $\begin{array}{c}p_{\max } \\
{[\%]}\end{array}$ & $\begin{array}{c}\lambda_{\max } \\
{[\AA]}\end{array}$ & $\begin{array}{c}\theta_{5500} \\
{[\mathrm{deg}]}\end{array}$ & $\begin{array}{c}\theta^{\prime} \\
{\left[10^{-3} \mathrm{deg} \AA^{-1}\right]}\end{array}$ \\
\hline HIP 20728 & $7.45 \pm 1.48$ & $134.2 \pm 26.7$ & $0.44 \pm 0.02$ & $4735 \pm 573$ & $167.9 \pm 0.9$ & $-1.2 \pm 0.9$ \\
HIP 20250 & $9.53 \pm 0.92$ & $104.9 \pm 10.1$ & $0.13 \pm 0.01$ & $5082 \pm 789$ & $3.8 \pm 1.8$ & $-1.7 \pm 1.2$ \\
HIP 20262 & $4.91 \pm 1.06$ & $203.7 \pm 44.0$ & $1.30 \pm 0.01$ & $5250 \pm 173$ & $4.4 \pm 0.2$ & $-2.2 \pm 0.2$ \\
HIP 20324 & $4.62 \pm 0.97$ & $216.5 \pm 45.4$ & $2.93 \pm 0.01$ & $5752 \pm 35$ & $14.2 \pm 0.0$ & $0.1 \pm 0.0$ \\
HIP 20663 & $4.77 \pm 1.07$ & $209.6 \pm 47.0$ & $0.95 \pm 0.01$ & $5301 \pm 1099$ & $19.8 \pm 0.2$ & $-0.2 \pm 0.1$ \\
\hline RY Tauri (HIP 20387) Foreground & $7.49 \pm 2.18$ & $139_{-9}^{+10} a$ & $0.73 \pm 0.36$ & $5244 \pm 373$ & $6.0 \pm 12.0$ & $-1.0 \pm 1.0$ \\
\hline
\end{tabular}

a Distance to Taurus-Auriga complex by Bertout et al. (1999).

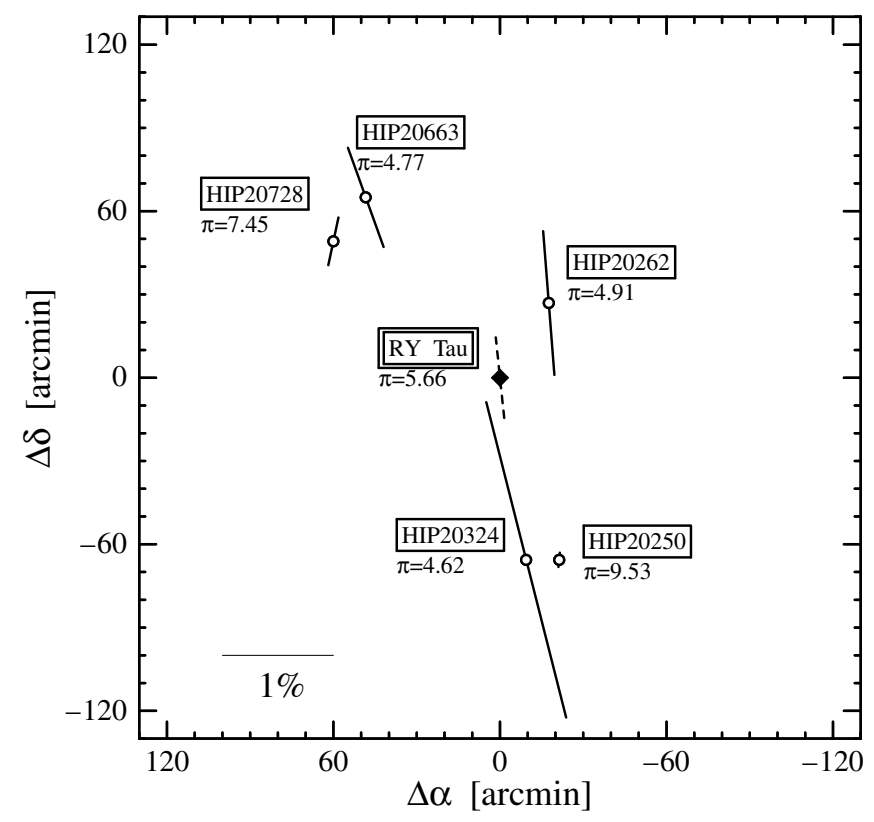

Fig. 3. Interstellar Polarization map around RY Tauri. Measured polarization of the field stars (solid lines) and estimated foreground polarization toward RY Tauri (dashed line) are shown. The line length indicates the polarization degree at polarization peak $\left(p_{\max }\right)$ and the direction indicates the position angle at $5500 \AA\left(\theta_{5500}\right)$.

We estimated the foreground polarization toward RY Tauri statistically from the interstellar polarization parameters measured toward these field stars. The position angles for the field stars are well aligned in the field (Fig. 3). We simply averaged all the position angles at $5500 \AA$ and estimated the position angle of polarization toward RY Tauri as $\theta_{5500}=6.0 \pm 12.0$ degrees. Similarly, the peak wavelength of the polarization curve and the dependency of position angle against wavelength were simply averaged over the field stars. They were determined as

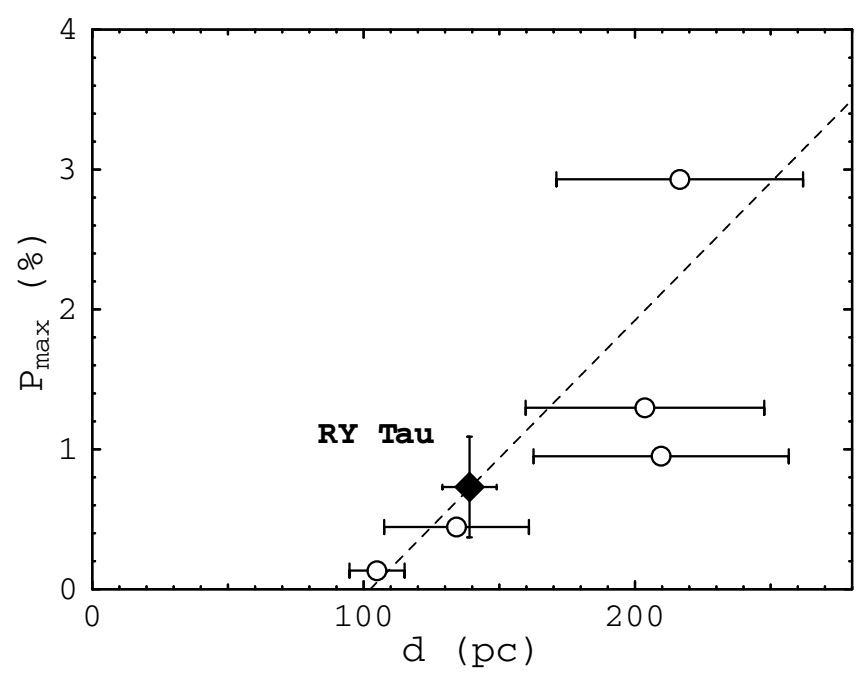

Fig. 4. The relation between interstellar polarization degree and distance around RY Tauri (open circles). The foreground polarization estimated at the distance of RY Tauri is also shown (filled diamond).

$\lambda_{\max }=5244 \pm 373 \AA$ and $\theta^{\prime}=(-1.0 \pm 1.0) \times 10^{-3}$ degrees $\AA^{-1}$, respectively. We found the increment of the peak polarization degree with distance as is shown in Fig. 4. Assuming a linear relation between $p_{\max }$ and the distance of a certain star $d$ as

$p_{\max }=p^{\prime} \cdot\left(d-d_{0}\right)$,

we derived $p^{\prime}=0.020 \pm 0.006$ percent $/ \mathrm{pc}$ and $d_{0}=102 \pm 11 \mathrm{pc}$. Since the distance to RY Tauri is estimated as $139_{-9}^{+10}$ pc by a statistical treatment of Hipparcos parallaxes of YSOs in TaurusAuriga complex (Bertout et al. 1999), we estimated foreground polarization degree toward RY Tauri to be $p_{\max }=0.73 \pm 0.36 \%$ from the relation of Eq. (7).

We summarize the estimated parameters on the bottom line of Table 3. We can now estimate the foreground polarization toward RY Tauri at an arbitrary wavelength using these parameters and assumed functions. 
Table 4. Measured polarization of forbidden emission lines and their nearby continuum.

\begin{tabular}{|c|c|c|c|c|c|c|c|}
\hline & \multirow{2}{*}{$\begin{array}{l}\text { Date } \\
(2002)\end{array}$} & \multirow{2}{*}{$\begin{array}{c}-E W \\
{[\AA]}\end{array}$} & \multicolumn{2}{|c|}{ Emission Line } & \multicolumn{2}{|c|}{ Nearby continuum } & \multirow{2}{*}{$\begin{array}{c}\text { Difference } \\
\Delta p_{\mathrm{i}-\mathrm{p}} \pm \sigma_{\Delta p_{\mathrm{i}-\mathrm{p}}}[\%]\end{array}$} \\
\hline & & & $q_{\text {integ }}[\%]$ & $u_{\text {integ }}[\%]$ & $q_{\mathrm{ph}}[\%]$ & $u_{\mathrm{ph}}[\%]$ & \\
\hline$[\mathrm{OI}] \lambda 5577$ & October 26 & $0.07 \pm 0.02$ & $-1.87 \pm 2.91$ & $-0.46 \pm 2.92$ & $2.47 \pm 0.02$ & $0.92 \pm 0.01$ & $4.6 \pm 3.2$ \\
\hline \multirow{3}{*}[\mathrm{OI}]{$\lambda 6300$} & March 1 & $0.75 \pm 0.08$ & $-0.66 \pm 1.03$ & $-0.30 \pm 1.03$ & $3.49 \pm 0.03$ & $0.31 \pm 0.03$ & $4.2 \pm 1.1$ \\
\hline & March 3 & $0.69 \pm 0.07$ & $-0.35 \pm 0.69$ & $-1.10 \pm 0.69$ & $3.81 \pm 0.02$ & $0.51 \pm 0.03$ & $4.5 \pm 0.8$ \\
\hline & October 26 & $0.50 \pm 0.04$ & $0.24 \pm 0.54$ & $-0.38 \pm 0.54$ & $2.92 \pm 0.01$ & $0.98 \pm 0.01$ & $3.0 \pm 0.6$ \\
\hline \multirow[t]{3}{*}[\mathrm{OI}]{$\lambda 6364$} & March 1 & $0.16 \pm 0.07$ & $2.77 \pm 11.60$ & $4.26 \pm 11.04$ & $3.43 \pm 0.03$ & $0.34 \pm 0.04$ & $4.0 \pm 11.2$ \\
\hline & March 3 & $0.15 \pm 0.05$ & $3.14 \pm 8.80$ & $-8.17 \pm 8.81$ & $3.81 \pm 0.02$ & $0.52 \pm 0.04$ & $8.7 \pm 9.0$ \\
\hline & October 26 & $0.14 \pm 0.04$ & $-8.90 \pm 3.46$ & $-7.49 \pm 3.43$ & $2.93 \pm 0.01$ & $0.99 \pm 0.01$ & $14.6 \pm 4.8$ \\
\hline \multirow[t]{3}{*}[\mathrm{NII}]{$\lambda 6583$} & March 1 & $0.11 \pm 0.06$ & $9.40 \pm 8.84$ & $-9.70 \pm 8.46$ & $3.34 \pm 0.08$ & $0.66 \pm 0.18$ & $12.0 \pm 10.2$ \\
\hline & March 3 & $0.11 \pm 0.04$ & $-3.85 \pm 10.34$ & $9.86 \pm 10.55$ & $4.04 \pm 0.07$ & $0.15 \pm 0.06$ & $12.5 \pm 10.9$ \\
\hline & October 26 & 0 & $\cdots$ & $\cdots$ & & & $\cdots$ \\
\hline \multirow[t]{3}{*}[\mathrm{SII}]{$\lambda 6716$} & March 1 & $0.04 \pm 0.03$ & $4.43 \pm 14.76$ & $-0.75 \pm 15.92$ & $3.43 \pm 0.02$ & $0.43 \pm 0.02$ & $1.5 \pm 15.5$ \\
\hline & March 3 & $0.04 \pm 0.02$ & $-15.05 \pm 10.30$ & $4.44 \pm 8.64$ & $3.95 \pm 0.01$ & $0.31 \pm 0.02$ & $19.4 \pm 10.7$ \\
\hline & October 26 & $0.04 \pm 0.01$ & $9.55 \pm 6.64$ & $2.42 \pm 6.54$ & $3.21 \pm 0.01$ & $1.04 \pm 0.01$ & $6.5 \pm 7.0$ \\
\hline \multirow[t]{3}{*}[\mathrm{SII}]{$\lambda 6731$} & March 1 & $0.16 \pm 0.05$ & $7.98 \pm 5.86$ & $2.86 \pm 5.92$ & $3.43 \pm 0.03$ & $0.43 \pm 0.03$ & $5.2 \pm 6.0$ \\
\hline & March 3 & $0.14 \pm 0.04$ & $0.06 \pm 3.91$ & $1.80 \pm 3.92$ & $3.95 \pm 0.02$ & $0.31 \pm 0.02$ & $4.2 \pm 4.1$ \\
\hline & October 26 & $0.07 \pm 0.02$ & $3.97 \pm 4.84$ & $-1.21 \pm 4.97$ & $3.21 \pm 0.01$ & $1.04 \pm 0.01$ & $2.4 \pm 5.1$ \\
\hline \multirow[t]{2}{*}[\mathrm{FeII}]{$\lambda 7155$} & March 1 & $0.18 \pm 0.07$ & $-0.45 \pm 5.90$ & $-1.10 \pm 5.99$ & $3.71 \pm 0.03$ & $0.39 \pm 0.05$ & $4.4 \pm 6.1$ \\
\hline & March 3 & $0.16 \pm 0.05$ & $7.34 \pm 4.46$ & $1.06 \pm 4.36$ & $3.93 \pm 0.03$ & $0.32 \pm 0.03$ & $3.5 \pm 4.7$ \\
\hline
\end{tabular}

\section{Results}

\subsection{Intensity spectra of identified forbidden lines}

Forbidden lines in T Tauri stars are generally thought to form in jets and winds around the central star (e.g., Kwan 1997). Each forbidden line generally consists of two components having different velocities: a low-velocity component (LVC) with a peak velocity blue-shifted in the range between $0 \mathrm{~km} \mathrm{~s}^{-1}$ and about $50 \mathrm{~km} \mathrm{~s}^{-1}$, and a high-velocity component (HVC) with that blueshifted by $-100 \mathrm{~km} \mathrm{~s}^{-1}$ or more.

The intensity spectra of the identified forbidden lines are shown in Fig. 2. The [OI] $\lambda 5577$ line is very weak but marginally indicates the presence of only a sharp LVC at the rest velocity. The [OI] $\lambda \lambda 6300$ and 6364 lines show both an LVC peak around the rest velocity and the wing blue-shifted by -100 to $-200 \mathrm{~km} \mathrm{~s}^{-1}$ which might be an HVC. The [SII] $\lambda \lambda 6716$ and 6731 lines still show emission at rest velocity but the centroid of the line seems more blue-shifted than that of the [OI] lines. The [NII] $\lambda 6583$ line indicates only an HVC with a peak velocity around $-100 \mathrm{~km} \mathrm{~s}^{-1}$. This tendency of velocity profiles for various kinds of FELs is consistent with the previous general understandings on FELs of TTSs (Hirth et al. 1997; Kwan 1997).

\subsection{Linear polarization in forbidden lines}

The $[\mathrm{OI}] \lambda 6300$ line is the strongest among the detected FELs and we found slight deviation in polarization within the line from the nearby continuum (Fig. 1), while most of the lines are too weak to recognize polarimetric features apparently in their polarization spectra. We integrated both the intensity and polarization spectra over the line profile as

$$
\begin{aligned}
I_{\text {integ }} & =\int_{\lambda_{\mathrm{c}}-\Delta \lambda / 2}^{\lambda_{\mathrm{c}}+\Delta \lambda / 2} I_{\text {line }}(\lambda) \mathrm{d} \lambda, \\
Q_{\text {integ }} & =\int_{\lambda_{\mathrm{c}}-\Delta \lambda / 2}^{\lambda_{\mathrm{c}}+\Delta \lambda / 2} Q_{\text {line }}(\lambda) \mathrm{d} \lambda, \\
U_{\text {integ }} & =\int_{\lambda_{\mathrm{c}}-\Delta \lambda / 2}^{\lambda_{\mathrm{c}}+\Delta \lambda / 2} U_{\text {line }}(\lambda) \mathrm{d} \lambda,
\end{aligned}
$$
in the object. In the theory of radiative transfer of polarized light,
(9) The foreground interstellar dust between RY Tauri system and us, aligned by magnetic field, acts as a polarizing modulator with dichroism, which modulates the initial polarization originating

and calculated the normalized Stokes parameters for the emission line by

$$
\begin{aligned}
& q_{\text {integ }}=Q_{\text {integ }} / I_{\text {integ }}, \\
& u_{\text {integ }}=U_{\text {integ }} / I_{\text {integ }},
\end{aligned}
$$

where the subscript "integ" represents the integrated value over the emission line. We took a peak wavelength of the intensity profile as $\lambda_{\mathrm{c}}$, the line center for integration. The full width of line integration $\Delta \lambda$ was determined with a criterion that the error in polarization degree of the integrated line becomes minimum. The derived values for the parameter $\Delta \lambda$ were typically about $1-$ $4 \AA$, where $50-70 \%$ of the whole line intensity is included in the integration. The integrated polarization of FELs and polarization of their nearby photospheric continuum are shown in Table 4.

A forbidden emission line is expected to show characteristic polarization differing from that at the nearby continuum in the case where the line photons take different paths and scattering geometries before reaching us than those of the continuum from the photosphere. To evaluate the statistical significance of polarization in the emission lines, we deduced the differential polarization vector $\left(\Delta q_{\mathrm{i}-\mathrm{p}}, \Delta u_{\mathrm{i}-\mathrm{p}}\right) \equiv\left(q_{\text {integ }}-q_{\mathrm{ph}}, u_{\text {integ }}-u_{\mathrm{ph}}\right)$, and its amplitude $\Delta p_{\mathrm{i}-\mathrm{p}} \equiv\left(\Delta q_{\mathrm{i}-\mathrm{p}}{ }^{2}+\Delta u_{\mathrm{i}-\mathrm{p}}{ }^{2}\right)^{1 / 2}$ and the error $\sigma_{\Delta p_{\text {i-p }}}$, which are also included in Table 4 . The polarization of the [OI] $\lambda 6300$ line was significantly differing from the nearby continuum at a $4 \sigma$ level $\left(\Delta p_{\mathrm{i}-\mathrm{p}}>4 \sigma_{\Delta p_{\text {i-p }}}\right)$. For the other FELs, we could not recognize statistically significant polarization differing from the continuum polarization within a polarimetric accuracy of 3-15 percent in the present study.

The measured polarization vectors of the $[\mathrm{OI}] \lambda 6300$ line at three epochs are consistent with each other within their errors (Table 4). We averaged these three vectors and obtained $\left(q_{[\mathrm{OI}] \mathrm{integ}}, u_{[\mathrm{OI}] \text { integ }}\right)=(-0.25 \pm 0.45,-0.59 \pm 0.45) \%$ as the [OI] $\lambda 6300$ polarization. Hereafter, we regard this average as the polarization of the $[\mathrm{OI}] \lambda 6300$ line.

\subsection{The intrinsic polarization of the [OI] 6300 line}




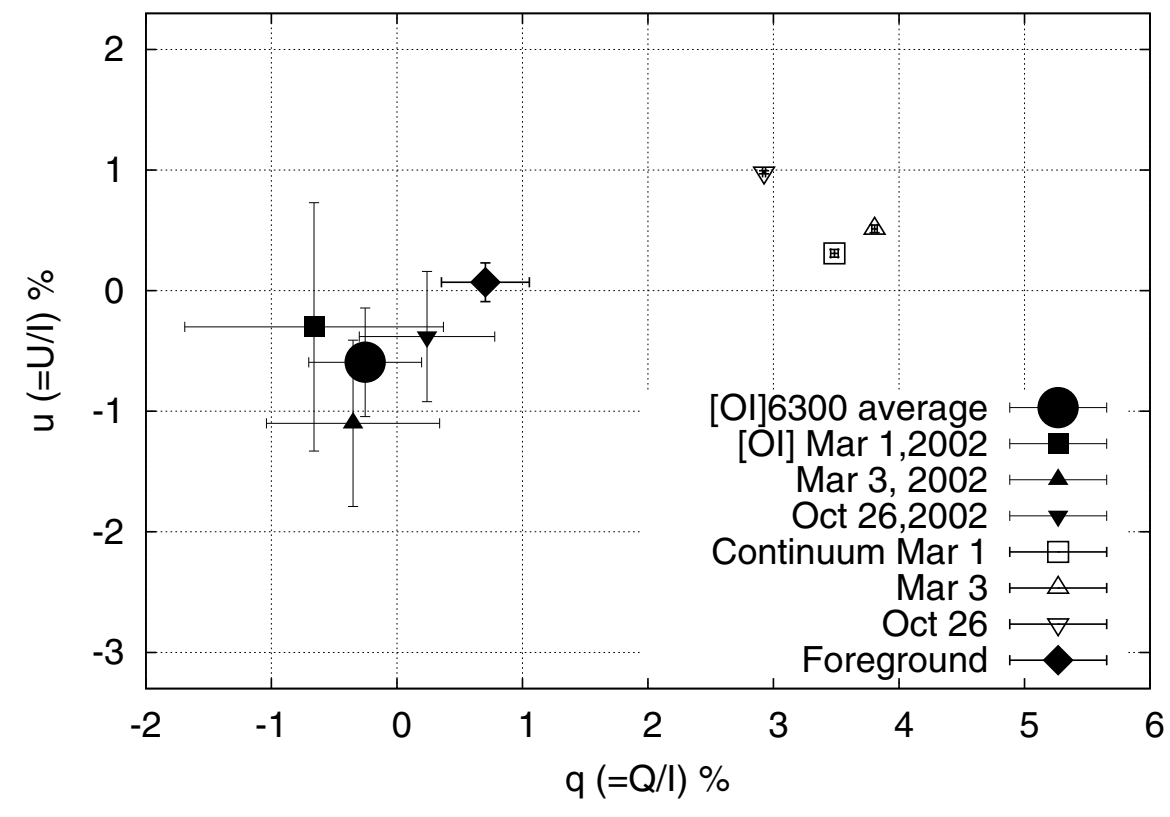

Fig. 5. Linear polarization of the $[\mathrm{OI}] \lambda 6300$ emission line at three observed epochs and their average are plotted in a $q-u$ plane. Continuum polarization at three epochs and estimated foreground polarization are also plotted. polarized light that has propagated through a dichroic medium is written as an incident Stokes vector multiplied by a Mueller matrix of the dichroic absorption. Assuming small amount of dichroism, which is appropriate for interstellar polarization with up to several percent, the modulation of polarization is approximately expressed as a simple vectorial addition of the normalized Stokes vector of the interstellar polarization to the incident normalized Stokes vector (Serkowski et al. 1962; Martin 1978).

Hence, the deduced polarization of the $[\mathrm{OI}] \lambda 6300$ line, $\left(q_{[\mathrm{OI}] \text { integ }}, u_{[\mathrm{OI}] \text { integ }}\right)$ in Sect. 3.2 , is not the true intrinsic polarization originating in the object but modified by foreground interstellar dust, namely $\left(q_{[\mathrm{OI}] \text { integ }}, u_{[\mathrm{O}] \text { integ }}\right)=\left(q_{[\mathrm{OI}]}, u_{[\mathrm{OI}]}\right)+$ $\left(q_{\mathrm{ISP}}, u_{\mathrm{ISP}}\right)$, here $\left(q_{[\mathrm{OI}]}, u_{[\mathrm{OI}]}\right)$ is an intrinsic normalized Stokes vector of the $[\mathrm{OI}] \lambda 6300$ line and $\left(q_{[\mathrm{ISP}]}, u_{[\mathrm{ISP}]}\right)$ is a normalized stokes vector of the foreground interstellar polarizaion.

Though the deduced vector is nearly unpolarized at first glance, it is chance cancellation between the intrinsic and foreground polarizations. This nearly zero polarization is consistent with the behavior of the observed polarization spectra shown in Fig. 1. If we measure a linear polarization spectrum of an unpolarized emission line overlapping a polarized continuum, as is the case for the observed $\left(q_{[\mathrm{O}][\text { integ }}, u_{[\mathrm{O}] \mathrm{I} \text { integ }}\right)$, we find dilution of the polarization degree across the line without any changes of the position angle. We can find the same behavior in Fig. 1.

The foreground interstellar polarization at $6300 \AA$ is estimated as $\left(q_{\mathrm{ISP}}, u_{\mathrm{ISP}}\right)=(0.71 \pm 0.35,0.07 \pm 0.16) \%$ from the parameters deduced in Sect. 2.4. In Fig. 5, the polarization of the [OI] $\lambda 6300$ line and that of the nearby continuum at three epochs, the average of three [OI] $\lambda 6300$ measurements, and the estimated foreground polarization are ploted on the normalized Stokes q-u plane. The apparent distinction between the observed [OI] $\lambda 6300$ polarization and the estimated foreground polarization, which is clearly seen in Fig. 5, strongly suggests that the [OI] $\lambda 6300$ line has significant intrinsic polarization originating in the RY Tauri system.

To evaluate the intrinsic polarization of the [OI] line, the foreground interstellar polarization vector should be subtracted from the observed polarization vector:

$\left(q_{[\mathrm{OI}]}, u_{[\mathrm{OI}]}\right)=\left(q_{[\mathrm{OI}] \text { integ }}, u_{[\mathrm{OI}] \text { integ }}\right)-\left(q_{\mathrm{ISP}}, u_{\mathrm{ISP}}\right)$.
Finally, the intrinsic polarization of the $[\mathrm{OI}] \lambda 6300$ line originating in the RY Tauri sytem was determined as $\left(q_{[\mathrm{OI}]}, u_{[\mathrm{OI}]}\right)=$ $(-0.96 \pm 0.57,-0.66 \pm 0.48) \%$, or $p_{[\mathrm{OI}]}=1.17 \pm 0.54 \%$ and $\theta_{[\mathrm{OI}]}=107^{\circ} \pm 13^{\circ}$. This is the first detection of significant linear polarization in FELs residing in TTSs. The intrinsic polarizations of the nearby continuum at three epochs are also deduced in the same way as $p_{\text {cont }}=2.4-3.1 \%$ and $\theta_{\text {cont }}=2^{\circ}-11^{\circ}$, varying with the epoch. These intrinsic polarization values are summarized in Table 5.

\section{Discussion}

The intrinsic polarization of the $[\mathrm{OI}] \lambda 6300$ emission line in RY Tauri and that of the nearby continuum differ significantly. The significant and distinct polarization of the [OI] emission indicates that the scattering geometry of the $[\mathrm{OI}]$ photons is different from that of the continuum photons. Therefore the detected polarization properties can be an important clue to clarify the distribution of $[\mathrm{OI}]$ emitting region and the surrounding material.

\section{1. [OI] 6300 emitting region and circumstellar structures}

The position angle (PA) of the long-axis of the circumstellar disk around RY Tauri was estimated as $27^{\circ} \pm 7^{\circ}$ by Kitamura et al. (2002) who presented the radio mapping observation at 2-mm continuum for the possible circumstellar disk of RY Tauri. The velocity gradient at $\mathrm{PA}=21^{\circ}$ detected from the $\mathrm{CO}(J=2-1)$ emission map (Koerner \& Sargent 1995) supports this estimation. The $\mathrm{H} \alpha$ jet at $\mathrm{PA}=295^{\circ}$ and its counter jet, which are perpendicular to the estimated disk position angle, were also found by St-Onge \& Bastien (2008). In Fig. 6, we show the position angles of the disk long-axis and jets, and those of the [OI] polarization and continuum polarization from our measurement, projected on the sky.

The position angle of the $[\mathrm{OI}] \lambda 6300$ polarization was $107^{\circ} \pm$ $13^{\circ}$, which is almost perpendicular to the disk position angle. Their difference $(\Delta \theta)$ was $80^{\circ} \pm 15^{\circ}$. It is also just parallel to the $\mathrm{H} \alpha$ jets $\left(\Delta \theta=8^{\circ} \pm 13^{\circ}\right)$. Both the perpendicularity and parallelism between the observed polarization and the 
Table 5. Intrinsic polarization of the $[\mathrm{OI}] \lambda 6300$ emission line and its nearby continuum.

\begin{tabular}{cccccc}
\hline \hline Band & Date & $q[\%]$ & $u[\%]$ & $p[\%]$ & $\theta[\mathrm{deg}]$ \\
\hline$[$ OI] $]$ 6300 & average over 3 nights & $-0.96 \pm 0.57$ & $-0.66 \pm 0.48$ & $1.17 \pm 0.54$ & $107 \pm 13$ \\
continuum & March 1, 2002 & $2.78 \pm 0.35$ & $0.24 \pm 0.16$ & $2.79 \pm 0.35$ & $2 \pm 2$ \\
& March 3, 2002 & $3.10 \pm 0.35$ & $0.44 \pm 0.16$ & $3.13 \pm 0.35$ & $4 \pm 2$ \\
& October 26, 2002 & $2.22 \pm 0.35$ & $0.91 \pm 0.16$ & $2.40 \pm 0.33$ & $11 \pm 2$ \\
\hline
\end{tabular}

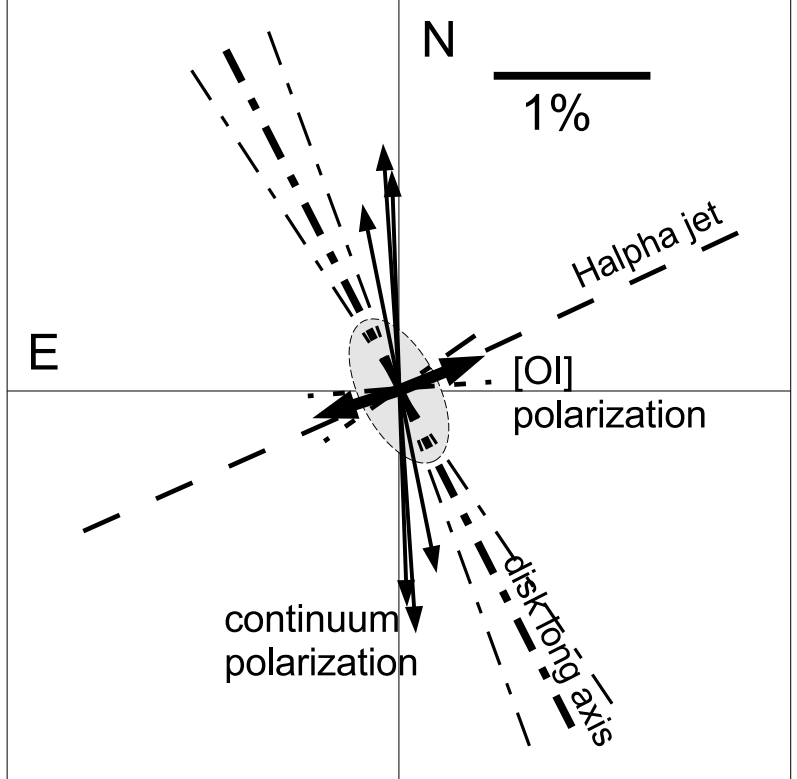

Fig. 6. The $[\mathrm{OI}] \lambda 6300$ polarization (thick arrow) with its error and the continuum polarization observed at three epochs (thin arrows) on the sky. The $\mathrm{H} \alpha$ jet position angle (St-Onge \& Bastien 2008) and the disk position angle with arbitrarily scaled inclined disk (Kitamura et al. 2002) are also indicated.

surrounding structures suggest that the $[\mathrm{OI}]$ polarization is well correlated with the axisymmetric circumstellar structures in the RY Tauri system. The [OI] $\lambda 6300$ emission line in TTSs is generally thought to be formed in the winds above the central region of the circumstellar disk for LVCs and in highly collimated jets on the axis of symmetry of the system for HVCs. It seems natural to consider that the gas in which [OI] photons are emitted is distributed well axisymmetrically around the central star, as are collimated jets or winds around the axis of the system, thus these photons are scattered by the axisymmetric circumstellar structures such as the disk and envelope.

The continuum polarization showed position angles between $2^{\circ}$ and $11^{\circ}$ varying with the epoch. The position angle seems roughly parallel to the disk position angle $\left(\Delta \theta=16-25^{\circ}\right)$. This parallelism between the continuum polarization and the disk long-axis might indicate that the surrounding envelope mainly scatters the continuum photons as Whitney \& Hartmann (1992, 1993) indicated in their light scattering calculations for young stellar objects with circumstellar scatterers. However, the continuum polarization showed slight but significant variation of polarization within only a few days, as has often been observed since the pioneering polarimetric work for this object (Bastien \& Landstreet 1979). The continuum polarization seems to be affected not only by the large scale structure such as an envelope but by a rapidly varying circumstellar structure near the central star as we can see in the daily variation of the polarization. The scattering geometry of the continuum emission may be actually more complicated.

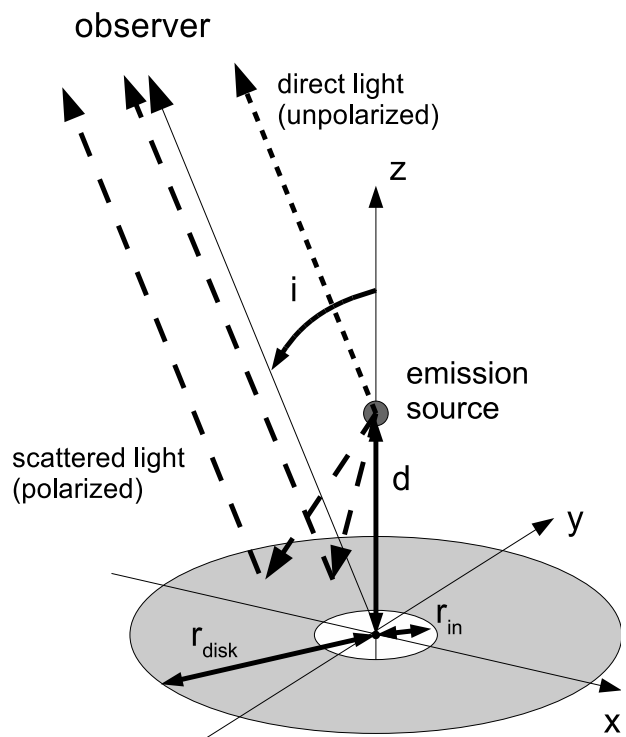

Fig. 7. The geometry of the simple light scattering model.

\subsection{Comparison with a simple scattering model}

The polarization of the $[\mathrm{OI}] \lambda 6300$ line is expected to reflect the geometrical configuration of the $[\mathrm{OI}]$ emitting regions and the circumstellar structure which scatters the [OI] photons. We try to estimate the position of the [OI] emitting region by comparing the observed polarization with a simple light scattering model.

The model geometry is described in Fig. 7. For simplicity, we considered that the [OI] emission emerges at a point on the axis of symmetry of the RY Tauri system; we assumed that the source is located either on a collimated jet or within a compact region in the wind near the $z$-axis. The system has also an optically thick, geometrically thin flat disk, where the incident photons are scattered at a ratio of an effective albedo $w_{\text {eff }}$. The disk has an inner hole with a radius of $r_{\text {in }}$, where no scattering of the incident photons occurs. The system is inclined toward the observer at an angle $i$. Photons from the [OI] emitting gas at a distance $d$ from the central star reach the observer either directly or after being scattered by the disk. Hence, the observed polarization consists of their composite. The reference coordinate of polarization is taken so that the apparent long-axis direction of the disk coincides with the positive Stokes- $q$ direction. The length scale in the model is measured by a unit of the disk radius $r_{\text {disk. }}$. The continuum radiation from the central star is not considered here because we treat only a forbidden line polarization completely separated from the continuum. We set the effective albedo of the disk $w_{\text {eff }}$ to 0.5 as a trial. We applied the scattering phase functions of Rayleigh scattering and of MRN dust mixtures at $6300 \AA$ calculated by White (1979) to take into account the size distribution of dust particles. We finally calculated the emerged polarization degree $q \equiv Q / I$ as a function of an [OI] source distance $d$ for a number of inclination angles in the range of $i=15-75^{\circ}$ with 

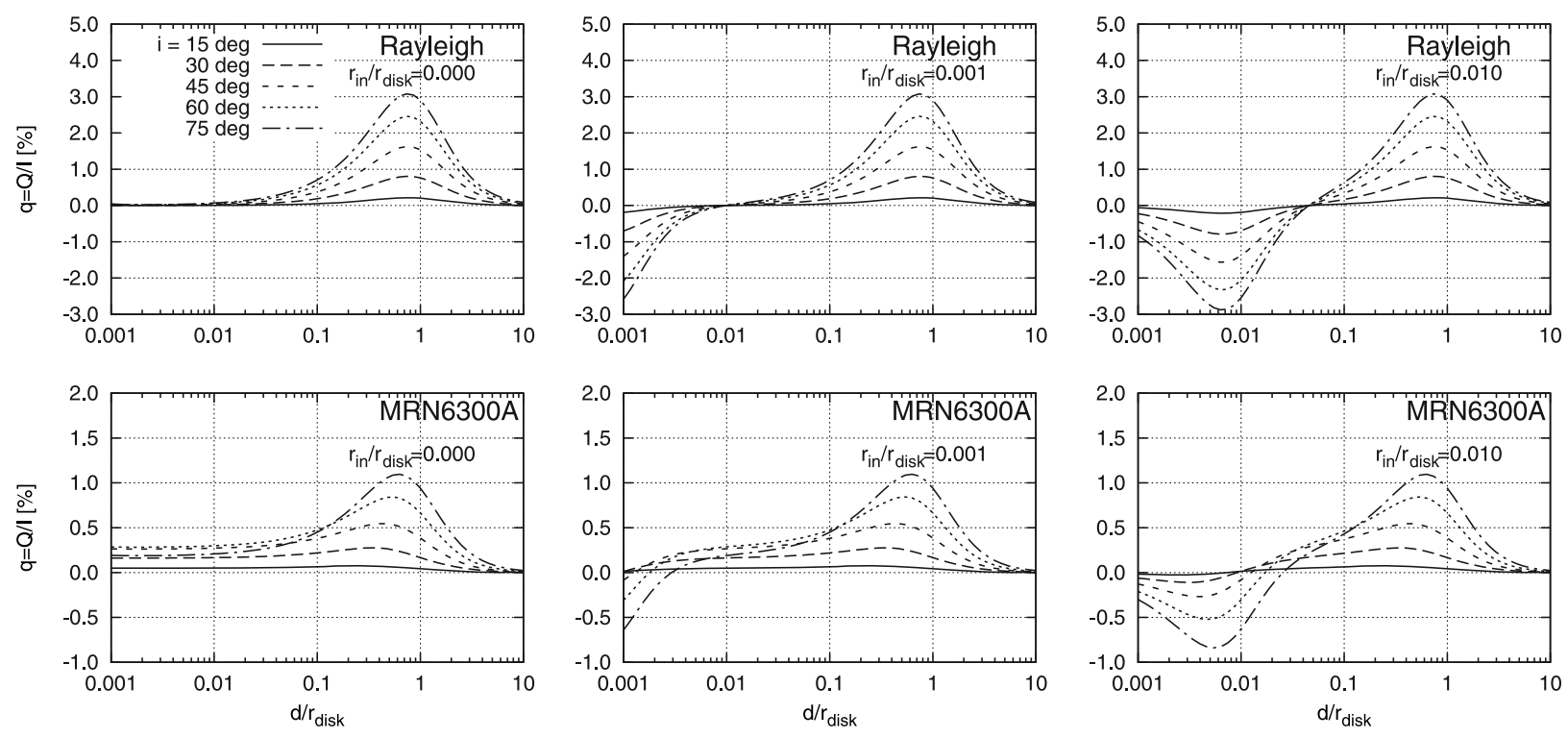

Fig. 8. The model calculations of linear polarization from a point source on a jet axis scattered by a flat disk with an inner hole. Polarization degree $q \equiv Q / I$ is shown against the source distance $d$ which is scaled by a disk radius $r_{\text {disk. }}$. Positive polarization is set in the direction of the disk long-axis. The calculations for various inclination angles of the disk between $15^{\circ}$ and $75^{\circ}$, and disk inner radii of $r_{\text {in }} / r_{\text {disk }}=0.000$ (without a hole), 0.001 , and 0.010 are shown. Rayleigh scattering (upper panels) and MRN dust scattering at $6300 \AA$ (lower panels) are considered as a scattering phase function.

disk inner hole radii of $r_{\text {in }} / r_{\text {disk }}=0.000,0.001$, and 0.010 , and two scattering phase functions. The results are shown in Fig. 8.

The behavior of the calculated polarization is qualitatively interpreted as below. At the beginning, we examine the results for Rayleigh scattering. In the calculations without a disk inner hole (the upper left panel of Fig. 8), only positive polarization appears with a peak at a moderate source distance. For small source distances, the source strongly illuminates the central portion of the disk, from which a large amount of scattered emission emerges. When we see the inclined disk system projected on the sky, the apparent centroid of the illuminated portion resides in the direction of the disk short axis from the source, thus positive polarization parallel to the disk long-axis emerges as a whole. As the source distance increases, the polarization degree of the sum of scattered light from the whole disk portions increases because directions of apparent scattering planes of all the scattered photons tend to converge. Meanwhile, the flux ratio of polarized scattered light from the disk to the constant unpolarized direct light from the source falls off with distance because the solid angle at which the source views the disk gets smaller. The behavior of the model polarization can be explained by compensation between these two effects. An increase in polarization with an inclination angle is explained by the growth of apparent asymmetry of the scattering geometry projected on the sky. The behavior changes significantly in the case with an inner hole (the upper panels of Fig. 8, $r_{\text {in }}=0.001$ and 0.010 cases). Negative polarization appears at small source distances, while the polarization becomes positive and approaches zero after a positive peak at larger distances as well as the results without an inner hole. For small distances, the scattered light from the annulus region around the inner hole dominates. As a result, negative polarization perpendicular to the disk long-axis emerges as a whole. For large distances, the solution asymptotically approaches the shape similar to that without an inner hole because global geometry approximately approaches that without a hole. In the MRN dust calculations, polarization tends to be smaller and its peak shifts to smaller source distances than the Rayleigh scattering calculations because the MRN dust tends to scatter incident photons into forward directions effectively.

Now we estimate the [OI] $\lambda 6300$ source distance $d$ applying the observed [OI] polarization and the other parameters of RY Tauri to the model. The inclination angle of the disk was estimated as $i=59^{\circ} \pm 7^{\circ}$, and the disk radius $r_{\text {disk }}$ was as $51 \mathrm{AU}$ from the 2-mm imaging (Kitamura et al. 2002). Akeson et al. (2005) performed near infrared interferometry and estimated the inner disk radius as $r_{\text {in }} \sim 0.2 \mathrm{AU}$ applying a ring model for the $K$-band continuum emitting region. The observed [OI] polarization perpendicular to the disk long-axis corresponds to $q \sim-1.17 \pm 0.54 \%$ on the coordinate of the current model. The results of calculations for the above parameters and two scattering phase functions are shown in Fig. 9 with the observed [OI] polarization.

We found some possible ranges of the source distance $d$ that satisfy both the model and observation. For the Rayleigh scattering case, the solutions are 0.25-0.51 AU and 0.02-0.06 AU. For the MRN dust case, the solution is $0.08-0.12 \mathrm{AU}$, though it is marginal. Such a precise comparison between the model calculation and observation, however, seems to be rarely useful because the model includes parameters with rough estimation such as an effective albedo $w_{\text {eff }}$. On the other hand, irrespective of roughness of these estimations, the general tendency of the model calculation that shows negative polarization for small source distances does not change. The model calculations show negative polarization with a substantial amplitude at source distances less than a few $\times 0.1$ AU. Here, we tentatively conclude that the $[\mathrm{OI}] \lambda 6300$ emitting region resides roughly within a few $\times 0.1$ AU from the central star.

Our result is consistent with the general understandings of upper limits of the [OI] $\lambda 6300$ emitting region. Hirth et al. (1997) showed that $[\mathrm{OI}] \lambda 6300$ emitting region for TTSs in Taurus cloud resides within $0.1^{\prime \prime}$ from the central star for LVCs and within $0.2^{\prime \prime}$ for HVCs, which correspond to a few $\times 10$ AU or less in projected distance, by seeing limited long slit spectroscopy. However, more precise position of the [OI] emitting region 


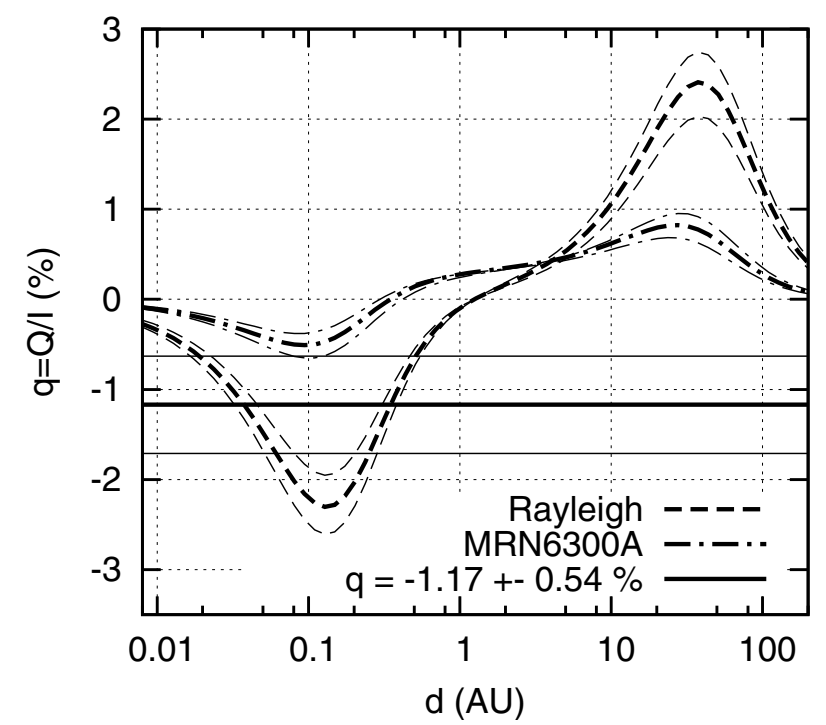

Fig. 9. Model calculations for RY Tauri $\left(i=59^{\circ} \pm 7^{\circ}, r_{\text {disk }}=51 \mathrm{AU}\right.$, and $\left.r_{\text {in }} \sim 0.2 \mathrm{AU}\right)$. The polarization $q \equiv Q / I$ against the source separation $d$ for Rayleigh scattering (dashed lines) and for MRN dust at $6300 \AA$ (chain lines) are shown with their errors. The observed [OI] $\lambda 6300$ polarization and its error are also indicated (horizontal solid lines).

beyond their resolution of $0.1^{\prime \prime}$ or $\sim 10$ AUs has not been revealed. Our result shed light on the issue by the spectropolarimetric approach. The measured [OI] polarization compared with the light scattering model indicates that a large amount of an [OI] $\lambda 6300$ line emerges in a very compact region in either a collimated jet or wind near the central axis, within a few $\times 0.1 \mathrm{AU}$ of the central star, and is scattered by a disk with an inner hole in the case of RY Tauri.

\subsection{Future prospects}

We achieved enough polarimetric accuracy only for an [OI] $\lambda 6300$ line among several FELs. However, polarization measurement of the other FELs emitted at a more distant position from a central star might help us to understand the polarization properties of FELs. Hirth et al. (1997) revealed that the spatial offset of [SII] emitting region of TTSs is 2-4 times larger than that of [OI], and that of [NII] lines is 1.5-2 times larger than [SII]. These lines are often observed as more distant jets or Herbig-Haro objects too. The polarization measurement of such lines for TTSs, whose emitting distance from the central star are reasonably well understood by long slit spectroscopy, will help us to evaluate the scattering models such as those we applied.

Polarization of an FEL that is emitted most distantly from the central star, such as [NII], might also be a reliable indicator of the foreground polarization. The intrinsic polarization of the line is expected to decrease and approach zero as the emitting region departs from the central star because scattered polarized flux decreases relative to the direct unpolarized light from the source as indicated by our model calculations (see Fig. 8). Thus the line shows only the foreground polarization when we measure its polarization. If this is the case, we acquire the powerful, independent method to estimate foreground polarization toward TTSs often difficult to estimate. Such an independent reliable estimation of the foreground polarization, which assigns the correct origin of polarization on a q-u plane, is crucial when we associate observed polarization with the geometry of the system quantitatively by use of intrinsic polarization spectra in the coordinate of polarization degree and position angle.

We detected intrinsic polarization for an $[\mathrm{OI}] \lambda 6300$ line but failed to measure velocity resolved polarization profile because of insufficient exposure, despite the sufficient wavelength resolution of the instrument. The deeper exposure at a wavelength resolution similar to that described in this work might reveal the polarimetric line profile for FELs. This enables us to separate the polarization of an LVC and an HVC, which are thought to have emerged from distinctly different regions, and gives us clearer information on the structure of jets and winds.

Polarization of FELs of TTSs are prospective as valuable probes for investigating the geometry of jets and winds near the central star. It is necessary to increase polarimetric measurements of FELs for a number of TTSs and various kinds of lines with deeper exposure.

\section{Summary}

We performed high-dispersion spectropolarimetry for the $\mathrm{T}$ Tauri star RY Tauri and measured linear polarization in seven forbidden lines [OI] $\lambda \lambda 5577,6300,6364,[\mathrm{NII}] \lambda 6583$, [SII] $\lambda \lambda 6716,6731$, and [FeII] $\lambda 7155$. This is the first highdispersion spectropolarimetric measurement for forbidden lines in T Tauri stars.

We successfully detected intrinsic polarization in the [OI] $\lambda 6300$ forbidden line. The intrinsic [OI] $\lambda 6300$ polarization after correction of the reliable independent estimation of the foreground polarization was derived as $p_{[\mathrm{OI}]}=1.17 \pm 0.54 \%$ and $\theta_{[\mathrm{OI}]}=107^{\circ} \pm 13^{\circ}$. The [OI $] \lambda 6300$ polarization apparently differs from the polarization of the nearby continuum derived as $p_{\text {cont }}=2.4-3.1 \%$ and $\theta_{\text {cont }}=2^{\circ}-11^{\circ}$. The significant intrinsic polarization of $[\mathrm{OI}] \lambda 6300$ indicates that the scattering geometry of [OI] $\lambda 6300$ photons is different from that of continuum photons emitted from the photosphere.

The position angle of [OI] $\lambda 6300$ polarization is compared with the circumstellar structures found by the previous studies. It is nearly perpendicular to the disk long-axis and parallel to the optical $\mathrm{H} \alpha$ jet. Both the perpendicularity and parallelism potentially suggest relatively axisymmetric distribution of the [OI] $\lambda 6300$ emitting region in a jet and/or winds and of scattering medium such as a disk and an envelope. We constructed a simplified scattering model with a point source as an [OI] emitter on a jet axis and a flat disk with an inner hole as a scatterer. The comparison between the model and observed polarization suggests that the $[\mathrm{OI}] \lambda 6300$ emission emerges close to the central star with a possible separation of less than a few $\times 0.1 \mathrm{AU}$.

To clarify the geometry and kinematics of jets and winds of $\mathrm{T}$ Tauri stars, measurement of linear polarization is one valuable method. However, significant measurement has been limited to only one emission line [OI] $\lambda 6300$ for only one object RY Tauri at present. It is necessary to increase the number of target $\mathrm{T}$ Tauri stars and kinds of forbidden lines for polarimetric measurements with reliable precision.

Acknowledgements. We are deeply grateful to Dr. R. Hirata for his valuable support and suggestions throughout the development of the spectropolarimeter LIPS and observation. We would like to thank Dr. A. Pickles and all the staff of the UH 2.2-m telescope for their great support among our observation. We also gratefully thank the staff of the Subaru Telescope for their kind support. We thank the referee for sincere comments and suggestions on the manuscript. Use of the UH 2.2-m telescope for the observations is supported by NAOJ. The development of LIPS is supported by the Subaru Telescope R\&D Committee. 


\section{References}

Akeson, R. L., Walker, C. H., Wood, K., et al. 2005, ApJ, 622, 440 Bacciotti, F., Mundt, R., Ray, T. P., et al. 2000, ApJ, 537, L49

Basri, G., Martin, E. L., \& Bertout, C. 1991, A\&A, 252, 625

Bastien, P., \& Landstreet, J. D. 1979, ApJ, 229, L137

Bertout, C., Robichon, N., \& Arenou, F. 1999, A\&A, 352, 574

Bouvier, J. 1990, AJ, 99, 946

ESA 1997, VizieR Online Data Catalog, 1239, 0

Hamann, F. 1994, ApJS, 93, 485

Harries, T. J., \& Howarth, I. D. 1996, A\&A, 310, 533

Hartigan, P., Edwards, S., \& Ghandour, L. 1995, ApJ, 452, 736

Hartmann, L., \& Stauffer, J. R. 1989, AJ, 97, 873

Hartmann, L., Hewett, R., Stahler, S., \& Mathieu, R. D. 1986, ApJ, 309, 275

Hirth, G. A., Mundt, R., \& Solf, J. 1994a, A\&A, 285, 929

Hirth, G. A., Mundt, R., Solf, J., \& Ray, T. P. 1994b, ApJ, 427, L99

Hirth, G. A., Mundt, R., \& Solf, J. 1997, A\&AS, 126, 437

Holtzman, J. A., Herbst, W., \& Booth, J. 1986, AJ, 92, 1387

Ikeda, Y., Akitaya, H., Matsuda, K., et al. 2003, Proc. SPIE, 4843, 437

Kawabata, K. S., Okazaki, A., Akitaya, H., et al. 1999, PASP, 111, 898

Kitamura, Y., Momose, M., Yokogawa, S., et al. 2002, ApJ, 581, 357

Koerner, D. W., \& Sargent, A. I. 1995, AJ, 109, 2138

Kwan, J. 1997, Herbig-Haro Flows and the Birth of Stars, 182, 443

Martin, P. G. 1978, Cosmic dust. Its impact on astronomy, Oxford Studies in Physics (Oxford: Clarendon Press)
Montes, D., Ramsey, L. W., \& Welty, A. D. 1999, ApJS, 123, 283

Petrov, P. P., Zajtseva, G. V., Efimov, Y. S., et al. 1999, A\&A, 341, 553

Pudritz, R. E., Ouyed, R., Fendt, C., \& Brandenburg, A. 2007, Protostars and Planets V, 277

Pyo, T.-S., Kobayashi, N., Hayashi, M., et al. 2003, ApJ, 590, 340

Pyo, T.-S., Hayashi, M., Kobayashi, N., et al. 2006, ApJ, 649, 836

Ray, T., Dougados, C., Bacciotti, F., et al. 2007, Protostars and Planets V, 231

Samoylov, A. V., Samoylov, V. S., Vidmachenko, A. P., \& Perekhod, A. V. 2004,

J. Quant. Spectrosc. Radiat. Transf., 88, 319

Schmidt, G. D., Elston, R., \& Lupie, O. L. 1992, AJ, 104, 1563

Serkowski, K. 1962, Adv. Astron. Astrophys., 1, 289

Serkowski, K., Mathewson, D. L., \& Ford, V. L. 1975, ApJ, 196, 261

Shang, H., Li, Z.-Y., \& Hirano, N. 2007, Protostars and Planets V, 261

St-Onge, G., \& Bastien, P. 2008, ApJ, 674, 1032

Takami, M., Bailey, J., Gledhill, T. M., et al. 2001, MNRAS, 323, 177

Tody, D. 1993, Astronomical Data Analysis Software and Systems II, ASP Conf. Ser., 52, 173

Vink, J. S., Drew, J. E., Harries, T. J., et al. 2003, A\&A, 406, 703

Vink, J. S., Drew, J. E., Harries, T. J., et al. 2005, MNRAS, 359, 1049

White, R. L. 1979, ApJ, 229, 954

Whitney, B. A., \& Hartmann, L. 1992, ApJ, 395, 529

Whitney, B. A., \& Hartmann, L. 1993, ApJ, 402, 605

Whittet, D. C. B., Martin, P. G., Hough, J. H., et al. 1992, ApJ, 386, 562 